\title{
EL TRATAMIENTO DEL ERROR DESDE LA PERSPECTIVA DEL ALUMNO Y SU IMPACTO EN LA ENSEÑANZA- APRENDIZAJE DE EXPRESIÓN ESCRITA EN L2
}

\author{
Susana Merino Mañueco
}

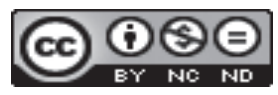

Esta obra está bajo una licencia Creative Commons Reconocimiento-No Comercial-Sin Obra Derivada 



\title{
EL TRATAMIENTO DEL ERROR DESDE LA PERSPECTIVA DEL ALUMNO Y SU IMPACTO EN LA ENSEÑANZA- APRENDIZAJE DE EXPRESIÓN ESCRITA EN L2
}

\author{
ERROR TREATMENT FROM STUDENT'S PERSPECTIVE. THE \\ IMPACT IN TEACHING AND LEARNING WRITING IN L2
}

\author{
Susana Merino Mañueco
}

\begin{abstract}
RESUMEN
El tratamiento del error en la enseñanza-aprendizaje de segundas lenguas (L2) ha sido entendido tradicionalmente como la rectificación de ciertas desviaciones de la norma encontradas en las producciones lingüísticas de los alumnos que estudian esa L2. Sin embargo, hoy en día lo entendemos como un concepto amplio que engloba una serie de componentes como son la detección, aceptación, corrección, etc. Los conceptos de error, corrección y feedback (como parte esencial del proceso de corrección de errores) han despertado el interés y la dedicación de profesores, lingüistas y especialistas en Didáctica y, en este sentido, se han llevado a cabo numerosos estudios en torno a estos. En el presente artículo nos adentramos en los procesos relacionados con el tratamiento de errores como son la corrección y la posterior dotación de feedback desde la perspectiva del alumno de L2 focalizando nuestra atención en los conocimientos, la aptitud y la actitud de este hacia el tratamiento de errores en expresión escrita.
\end{abstract}

Palabras clave: tratamiento del error, enseñanza-aprendizaje, L2, feedback, expresión escrita.

\begin{abstract}
Error treatment in L2 teaching and learning processes has been traditionally considered as the rectification to a deviation of standard rules found in L2 student's linguistic productions. Nowadays, we recognize it as a global concept that encompasses a series of related components: detection, acceptance and correction among others. The concepts of error, error correction and feedback (as an important part of error correction process) are receiving constant attention and action from teachers, linguists and specialists in Didactics. They all have been considered a focus of attention and they have been included in the second language (L2) teaching-learning process. The purpose of this study is to identify correction and feedback as processes related to error treatment from the L2 student's perspective focusing our attention in the knowledge, the abilities and the attitudes they have towards error treatment in L2 writing composition.

Key words: error treatment, teaching-learning, L2, feedback, writing composition.
\end{abstract}

\footnotetext{
Mtr. Susana Merino Mañueco. Doctoranda en Español: Lingüística, Literatura y Comunicación. Universidad de Valladolid. España.

Correo electrónico: susana.merino.manueco@uva.es
}

Recepción: 05- 12- 2017

Aceptación: 20- 02- 2018 


\section{Introducción}

Tanto profesores como especialistas en Lingüística y en Didáctica reconocen la importancia de la corrección y, consecuentemente, de la presencia del error en la enseñanzaaprendizaje de L2. Dicha importancia reside en concienciar tanto al alumno como al profesor en la incidencia que tiene el cómo, el cuándo y el qué se corrige en las producciones escritas de los alumnos de L2.

Durán (2011, p. 62), refiriéndose a la corrección de errores, considera que se trata de uno de los temas más controvertidos del área de enseñanza de L2, puesto que esta implica la presencia de una serie de factores personales, estrechamente relacionados con el profesor; lo cual le determina a considerarla como "a highly personal and complex issue".

La corrección de errores tiene una doble función instrumental; fuente de aprendizaje para el alumno al tiempo que se convierte en un "instrumento muy eficaz para la labor docente" (d'Aquino, 2016, p. 12). Así es como, por su función y por los numerosos factores personales que intervienen en la corrección de errores, nos encontramos ante una tarea de gran complejidad que implica un alto nivel de responsabilidad docente.

En el presente artículo nos adentramos en los procesos relacionados con el tratamiento de errores como son la corrección y la posterior dotación de feedback desde la perspectiva del alumno de L2 focalizando nuestra atención en los conocimientos, la aptitud y la actitud de este hacia el tratamiento de errores en expresión escrita.

\section{La corrección de errores como responsabilidad docente}

Tanto la corrección como el tratamiento de los errores son Actos Profesionales Docentes (APDs) asumidos por el Agente recurso humano-profesor (Dumbrăvescu, 2017; Guillén-Díaz y Castro-Prieto, 1998; Roy, 1991). Dichos APDs, como actividades que forman parte de la intervención didáctica y la evaluación del alumno, son objeto de toma de decisiones en la enseñanza efectiva. La detección de errores, su corrección y tratamiento pueden ofrecer solución a problemas concretos que han surgido en el aprendizaje causados, según encontramos expresado en d'Aquino (2016), por varios factores, entre estos, las dificultades propias de la lengua, interferencias con la lengua materna, interferencias con otras lenguas, aspectos emocionales y afectivos del alumno, procedencia lingüística y cultural, etc.

Según planteaba recientemente d'Aquino (2016, pp. 10-11), la corrección puede entenderse como "acto comunicativo" o "acto de comunicación" que se lleva a cabo con un único objetivo: "ayudar". En este acto entran en juego varios factores como la relación alumnoprofesor, la jerarquía de conocimientos, la edad del alumno, la actitud del profesor y del alumno ante el error, el tono que emplea el profesor y la retroalimentación que deben tenerse en cuenta a la hora de corregir errores. Una corrección entendida en términos de "interacción" puede llegar a adquirir una serie de connotaciones desde el punto de vista afectivo. Es el caso del llamado exceso de correcciones que puede convertirse en una fuente de frustraciones tanto para el profesor, como para el alumno.

En relación con los comentarios que añadimos a las redacciones de nuestros alumnos, partimos de la idea de Cassany (1993, pp. 11-12), quien indicaba que existen numerosas cuestiones sin resolver para el docente relacionadas con estos, a saber: "¿son realmente apropiados y provechosos [...], hacemos demasiados o demasiado pocos?”, “ilos entienden los alumnos?”, “ipor qué estos, al rehacer el texto, sólo aprovechan algunos comentarios y 
olvidan otros?”, o “¿por qué muchas veces la corrección fracasa y el alumno repite una y otra vez las mismas faltas?". Como conclusión a todas estas cuestiones, Cassany (1993) afirmaba que "sabemos muy poco o nada de todo esto" a lo que añade que "la corrección requiere urgentemente el interés y la dedicación científica de maestros y pedagogos". En su obra, Cassany (1993, p. 12) hace referencia a varios estudios realizados en castellano y catalán, por varios didactas y profesionales de la Linguíística, cuyas aportaciones, en palabras del propio autor, "son más útiles para los evaluadores [...] que para los maestros preocupados por buscar la forma más clara y eficaz de corregir las redacciones de los alumnos".

Por su parte, Fernández-López (1995, p. 212), refiriéndose a la reacción del profesorcorrector ante las producciones escritas de los alumnos, destacaba que "lo primero es reaccionar como interlocutores, no como correctores, porque distorsionamos el mismo proceso de aprender a escribir en la L2"; asimismo, "podemos proponer una corrección, lo más interactiva posible - de forma que el alumno tenga posibilidad de plantearse la duda, buscar, etc.- y centrada en lo que se está trabajando". Además, la citada autora insiste en que, en numerosas ocasiones, "si el aprendiz está intentando comunicar algo, con la atención centrada en el sentido, la corrección le pasa inadvertida", por lo que propone como solución "practicar la «corrección comunicativa»: si somos uno más en la interacción, podemos intervenir como lo hacemos en una conversación normal, dando la palabra que falta, retomando una frase, resumiendo una postura, etc." concediendo al alumno "la oportunidad de oír de forma correcta una determinada estructura" sin interrumpir la comunicación.

Truscott (1996), a su vez, aludía a otro inconveniente que entraña la corrección como parte de la responsabilidad del docente: la cantidad de tiempo que el profesor debe emplear en las correcciones. Se trata, según apunta el autor, de una cantidad de tiempo desmesurada, sobre todo cuando se trata de un grupo de alumnos numeroso, tal y como queda expresado a continuación:

The time problem is even greater -in fact, much greater-for teachers. In a class with many students and many writing assignments, correction of grammar errors can absorb an enormous amount of a teacher's time, time that could be spent more productively (and perhaps more pleasantly) on other things. (Truscott, 1996, p. 355)

También Cassany (1993, p. 11), recordando el estudio que hizo Sommers, en 1982, destacaba que la corrección de errores es "una de las tareas que consume más tiempo y esfuerzos del profesor". A todo esto, se añade el hecho de que dicha tarea, como resalta d'Aquino (2016, p. 7), debe realizarse "de una manera respetuosa hacia el productor del error". Más aún, Cassany (1993) planteaba la necesidad de autorreflexión sobre el propio trabajo de corrección que realizan los profesores ya que, según él, no solo emplean una cantidad de tiempo excesiva en corregir, sino también en escribir una serie de comentarios que, en la mayoría de los casos, resultan prácticamente invisibles e inservibles para el alumno.

La corrección de errores logra alcanzar su objetivo en la medida en que esta puede integrarse en la enseñanza-aprendizaje de L2 para que el alumno llegue a conseguir la eficacia en la comunicación, mostrando una actitud de confianza en sí mismo y en el aprendizaje. Desde esta perspectiva de concienciación y de reflexión sobre la corrección, es más que evidente la necesidad de integrar el error en el proceso de enseñanza.

\section{Causas y criterios en torno a la aparición del error}

Son muchas las teorías que giran en torno a la actitud de alumnos y profesores ante el error. Desde el ámbito español, Fernández (1997, p. 261) apoyaba la idea de que el alumno 
debe "asumir el error como un elemento no sólo ineludible, sino también necesario para aprender una lengua". Por tanto, la aceptación del error "cambia la perspectiva, la valoración ante este fenómeno, la actitud del que aprende y la dinámica del aula”. Así pues, es interesante acercarnos a este como un posible fallo humano que puede generarse tanto por parte del alumno (por haber experimentado una falta de atención, un fallo de memoria, etc.), como por la ineficacia del profesor.

La actitud del alumno ante los errores se halla, de acuerdo con Fernández-López (1995), en directa relación con la edad. En el caso de los adultos, los errores se muestran como indicadores de una incapacidad o conducta negativa según explicaba:

\footnotetext{
Para el que aprende una lengua, los errores, en una gran mayoría, se sienten como una limitación que, en el caso de alumnos adultos, conlleva muchas veces un recorte de la comunicación. En el aula pueden provocar además una sensación de infantilismo, o de inhibición adulta ante el no quedar bien, ante la corrección pública de los posibles fallos. (Fernández-López, 1995, p. 204)
}

En el caso concreto de los niños, el error no tiene una carga tan negativa y no influye tan decisivamente a la hora de comunicarse en una L2. Al tratarse de edades tempranas, el alumno no tiene una conciencia tan desarrollada en cuanto al error y a su impacto.

En lo que concierne a las causas asociadas al error Giovannini, Martín-Peris, Rodríguez-Castilla y Simón-Blanco (1996, p. 13) ponían de manifiesto que "una causa frecuente de error es el estrés asociado al hecho de hablar una lengua extranjera de forma imperfecta, y a la posibilidad de que uno se sienta ridículo o rechazado por el grupo". Este estado de tensión tiene un impacto negativo, en opinión de los citados autores, y es lo que "nos lleva a tener muchas dudas y vacilaciones y a cometer errores en el momento de hablar".

Asimismo, Torijano-Pérez (2004,p. 17) también hablaba de este sentimiento de "ridículo o de impotencia" que puede desembocar en lo que él llama "la temida inhibición del estudiante" y que, normalmente trae consigo el "desinterés y el abandono del proceso". El autor destacaba que, el comportamiento de los estudiantes, ante el error, viene acompañado de un sentimiento de culpabilidad que los acerca a la creencia de que son ellos los responsables de evitar su presencia. Dicho comportamiento de miedo a cometer errores y a hacer el ridículo puede llegar a ser "uno de los factores más inhibidores en cualquier situación formal de aprendizaje".

Nos situamos en la línea de Fernández (1997, pp. 30-31) por cuanto que, en el aula de L2, no todos los errores han de ser corregidos y, desde esta consideración, la misma autora establecía una jerarquía en torno a la corrección de errores. En este sentido, la autora planteaba "los conceptos de «gramaticalidad» y de «aceptabilidad»". En su visión, "la gramaticalidad hace referencia a la conformidad de la producción con el sistema de la lengua que se aprende" al tiempo que "la aceptabilidad se somete al juicio del informante, que, en cada situación concreta, valora si el mensaje se entiende, es confuso, es adecuado a la situación: es, en una palabra, aceptable o no". Dichos conceptos resultan ser de gran importancia a la hora de determinar la gravedad de los errores por lo que: "desde una perspectiva comunicativa, el criterio prioritario es, evidentemente, el de la aceptabilidad. Un error será más o menos grave en la medida en que afecte al mensaje y dificulte o distorsione la comunicación". Se unen a los ya mencionados conceptos, por una parte, el nivel de competencia comunicativa en la L2 y, por otra, las necesidades lingüísticas de los aprendices. Como profesores, nos corresponde hacer ver a nuestros alumnos que "los primeros criterios que asumimos son los comunicativos: el logro o no de la comunicación y el mayor o menor grado de distorsión del mensaje". La importancia de dar a conocer el valor que otorgamos al intento de comunicación 
por parte del alumno reside en la necesidad de evitar que este experimente inhibición ante la producción en L2. Sin embargo, a pesar de los conceptos ya enumerados en torno al error, retenemos la idea de Fernández (1997) respecto a que "estos no son criterios objetivos, y aunque se elaboren escalas más o menos precisas, tal vez, como apuntábamos más arriba, no seamos los profesores, ni los estudiosos del tema, los árbitros más imparciales para dilucidar la efectividad comunicativa de los aprendices".

\section{Investigaciones previas en torno al error, la corrección y el correspondiente feedback en las producciones escritas de los alumnos de L2}

Los estudios de investigación centrados en la corrección de las producciones escritas de los alumnos de L2 se han venido sucediendo a lo largo de los años. Tomando como punto de partida la década de los años 80, nos disponemos a realizar un recorrido a través de una serie de trabajos de investigación llevados a cabo por diversos autores e investigadores vinculados al tema del tratamiento del error en la enseñanza-aprendizaje de L2.

Recurrimos a Zamel (1985, p. 80) quien destacaba que, teniendo en cuenta el grado de importancia de las respuestas de los profesores a las producciones escritas de los alumnos y la cantidad de tiempo y esfuerzo invertidos en ellas, resulta llamativo el hecho de que estas no hayan recibido hasta ahora la atención que merecen. En virtud de ello, insistía en la escasez de estudios empíricos orientados al análisis de dichas respuestas las cuales, en palabras de la autora, encierran significados subyacentes acerca de la naturaleza y las funciones de la expresión escrita. La mencionada autora, llevó a cabo un análisis de los comentarios escritos de 15 profesores en 105 textos de alumnos de ESL (English as a Second Language) intentando demostrar que dichos comentarios constituían una representación del comportamiento de los profesores informantes a la hora de responder a las producciones escritas en L2 de sus alumnos. El análisis puso de manifiesto que los profesores realizan una lectura superficial de las producciones de los alumnos, proporcionando comentarios contradictorios y correcciones inconsistentes y, además, responden a los textos como productos finales y no como borradores intermedios susceptibles de revisión. En palabras de la autora:

ESL writing teachers misread student texts, are inconsistent in their reactions, make arbitrary corrections, write contradictory comments, provide vague prescriptions, impose abstract rules and standards, respond to texts as fixed and final products, and rarely make content-specific comments or offer specific strategies for revising the text. (Zamel, 1985, pp. 85-86)

En consecuencia, la citada autora proponía un cambio de dirección hacia una modalidad de respuestas en las que se incluyan una serie de estrategias que indiquen al alumno la forma en que deben llevar a cabo la revisión y posterior corrección de sus producciones escritas. La autora sugiere, además, que sean los alumnos quienes asuman la responsabilidad sobre sus propias producciones partiendo de la elaboración de distintos borradores hasta llegar al producto final (Zamel, 1985).

Estudios como el de Zamel (1985) determinaron un cambio en el enfoque pedagógico en la enseñanza de producción de textos escritos en L2 dentro del contexto educativo norteamericano. Los profesores comenzaron a inclinarse por el uso de otros métodos de corrección y de feedback entre los que se encuentran las entrevistas personales entre profesor y alumno denominadas "one-to-one writing conferences" o "teacher-student writing conferences" (Ferris, 2003, p. 20), o las sesiones de revisión por parte de uno o varios 
compañeros de clase o "peer review sessions" (Ferris, 2003, p. 22), en las que se insta a otros alumnos del aula a colaborar con el autor de la producción escrita.

Respecto a los métodos, Zamel (1985, pp. 94-95) proponía utilizar la entrevista personal con los alumnos a partir de lo que ella denomina "cycles of revision" o borradores encadenados "so that students better understand how to revise their writing". Además, Campbell (1998, p. 3), añadía que es el profesor quien debe asumir el papel de guía que dirige a sus alumnos a través de los distintos borradores hasta conseguir el producto final. Estas posiciones se acercan al enfoque metodológico centrado en el proceso o producción del texto o "process-oriented pedagogy" al que Ferris y Hedgcock (1998, p. 197) hacen referencia afirmando que, a través de su aplicación en el aula, los alumnos podrán mejorar sus competencias en cuanto a revisión y creación de nuevas ideas. Además, el impacto de las nuevas tecnologías de la educación y su uso en el aula permiten desarrollar otras modalidades de aportar feedback, a saber: la grabación de los comentarios del profesor o "audiotaped feedback" (Ferris, 2003, p. 20), comentarios incluidos en el texto del alumno a través de un programa informático o incluso feedback enviado a través de correo electrónico o "email feedback" (Ferris, 2003, p. 20).

Dentro del contexto español, encontramos a Cassany (2009, p. 62), quien pone de manifiesto la existencia de dos técnicas de producción escrita alternativas en el aula de L2. En la primera, el profesor corrige el producto final del alumno sin que exista interacción oral entre ellos durante el proceso de producción. Así pues, profesor y alumno solo interactúan "antes y después de la composición", siendo el profesor quien asume el rol de autoridad a través del cual "dispone lo que se escribe al principio" y "juzga cómo se ha hecho al final". Esta técnica se concretiza en lo que conocemos como "product-oriented pedagogy" o enfoque centrado en el producto o texto final. En la segunda, el autor del texto elabora su producción escrita ayudado por el profesor y por el resto de sus compañeros de aula. Todos ellos "pueden colaborar con el autor durante todo o parte del proceso de composición y de modos sustancialmente distintos", interactuando con él de forma oral. Asimismo, "la actividad de producción textual se convierte en un conjunto variado de subtareas (planificación, textualización, etc.)" que el alumno lleva a cabo gracias a la cooperación de profesor y compañeros de aula. Dicha técnica corresponde al enfoque metodológico conocido como "process-oriented pedagogy" o enfoque centrado en el proceso de escritura.

La técnica de producción textual denominada "borradores encadenados", que mencionábamos anteriormente, se materializa en una serie de entrevistas entre el alumno (autor) y el profesor en las que se puede incluir la presencia de otros compañeros de aula que colaboran en el proceso de producción ayudando al autor en sus tareas de planificación, revisión, etc. El profesor solicita al alumno la elaboración de un primer borrador, a partir de un cuadro-resumen o esquema que incluya las ideas principales del texto. Después de elaborar este primer borrador, el alumno será instado a aproximarse a la mesa del profesor (el cual, puede encontrarse solo o acompañado de uno o varios alumnos) donde tendrá lugar la conferencia entre profesor y alumno-autor de la producción escrita o entre profesor, alumno-autor y alumno o alumnos colaboradores. Tras la escucha atenta de la lectura por parte del alumno de su primer borrador, el profesor procederá a proporcionar el feedback correspondiente; este puede ir acompañado de determinadas contribuciones del alumno o alumnos colaboradores. Además, este podrá concretarse en feedback oral: comentarios que el profesor proporcionará al alumno en los cuales incluirá no solo consejos y recomendaciones, sino también frases de apoyo y ánimo, gestos de complicidad y cercanía, etc., acompañado de feedback escrito, a saber: notas 
redactadas por el profesor durante el transcurso de la lectura. El alumno, habiendo recibido el feedback del profesor, puede volver a su escritorio para elaborar un nuevo borrador con la ayuda del feedback proporcionado. Este proceso se repetirá en varias ocasiones hasta que el alumno consiga el producto o borrador final deseado.

Con el fin de contribuir a un aumento en el nivel de autonomía del alumno, la técnica de "borradores encadenados" plantea la posibilidad de hacer al alumno partícipe del proceso de corrección, otorgándole un papel activo en dicho proceso. Es el propio alumno quien lleva a cabo las correcciones siguiendo las orientaciones del profesor y/o de los alumnos colaboradores. Por su parte, el profesor mostrará una actitud de cercanía y complicidad a través de sus gestos haciéndole consciente de su papel de orientador.

Se ha hablado mucho acerca del tipo de feedback que el profesor de L2 debe proporcionar en cada una de las etapas del proceso de escritura. Así pues, Fathman y Whalley (1990, pp. 186-187) realizaron un estudio a un grupo de alumnos de inglés como segunda lengua (ESL) a los que se les expuso ante distintos tipos de feedback. Analizaron el impacto de la corrección y posterior feedback de los errores gramaticales (o errores de forma) respecto a la corrección y posterior feedback de los errores de contenido concluyendo que los dos tipos de corrección contribuyen de una forma positiva a la mejora de la competencia de expresión escrita del alumno de L2. En este sentido, las mencionadas autoras señalaban que, tras la exposición de los alumnos ante la corrección gramatical y posterior feedback, estos experimentan una mejora significativa en su competencia gramatical o, recurriendo a las palabras de las propias autoras, "students showed significant improvement in grammatical accuracy". Por otra parte, respecto a la eficacia de la corrección del contenido y al correspondiente feedback en la mejora de la composición escrita subrayaban la importancia de los comentarios del profesor, dirigidos a sugerir revisiones y unidos a estímulos y palabras de apoyo de manera que: "general comments giving encouragement and suggesting revisions helped improve the content of composition rewrites". En su opinión, el feedback de forma y el feedback de contenido pueden aparecer de forma conjunta o independiente sin que al alumno pueda experimentar una sensación de sobrecarga. A partir del mencionado estudio, Fathman y Whalley (1990) pudieron concluir que la presencia o ausencia del feedback incide favorable o negativamente en la calidad de la composición escrita.

Años más tarde, Ferris (2003, p. 24) insistía en esta misma idea de que el feedback que acompaña a la corrección de las producciones escritas de los alumnos de L2 debe enfocarse tanto en el contenido como en la forma. Lo importante, según la autora, es que dicho feedback se presente adaptado a las características y a las necesidades específicas de cada alumno. Ha habido una sucesión de propuestas contrarias a la de Ferris (2003, p. 28), quien defendía el uso del feedback y sus beneficios en las producciones escritas de los alumnos. Así es como, dichas propuestas evidenciaban que las correcciones y los comentarios del profesor respecto a las composiciones escritas pueden causar más daño que beneficios. Por otro lado, la autora afirmaba que las investigaciones más recientes sobre el uso de técnicas de producción escrita, a través de varios borradores, demuestran que, sin duda alguna, el feedback del profesor ayuda a los alumnos en la mejora de la escritura o en palabras de la autora: "teacher feedback certainly can and often does help student writers to improve their writing from one draft to the next and over time" y, de acuerdo con esto, los propios alumnos hacen referencia a este como una herramienta de gran ayuda. A modo de conclusión, debemos destacar que los pocos estudios empíricos realizados en torno a este tema demuestran, en parte, la utilidad del feedback dentro 
del proceso de enseñanza-aprendizaje de L2 y la necesidad de su presencia en el aula, (Ferris, 2003; Ferris y Hedgcock, 1998; Gascoigne, 2004; Leki, 1990). El feedback queda, por tanto, definido como un proceso todavía desconocido, cuya incidencia se ha de verificar o, como afirmaba Ferris (2003, p. 41) "there is a lot to be learned about the best ways in which to give feedback and about how (and if) it facilitates students' writing development over time".

\section{El feedback y su proyección en la enseñanza-aprendizaje de L2}

Recurrimos al término feedback como sinónimo de retroalimentación, tal y como aparece en el Diccionario de términos clave de ELE (Centro Virtual Cervantes, 1997-2017) en su versión electrónica. Dichos términos son utilizados como sinónimos por autores como: de la Torre, S., Mallart, J., Tort, L1., Rajadell, N., Laffitte, R. y Oliver, C. (1994); García-Heras (2004); Jiménez-González y Muñetón-Ayala (2002); Jericó (2013); López-Hernández (2009) y Vargas-Venegas (2008), entre otros. Se observa, sin embargo, que hay un gran número de didactas y lingüistas que establecen una diferencia entre corrección y feedback, en el aula de L2, insistiendo en la idea de que, el segundo es un término mucho más amplio que el primero, llegando incluso a formar parte de este (Pawlak, 2014). Según el citado diccionario, el feedback o retroalimentación:

Consiste en el mecanismo mediante el cual el aprendiente de L2 incorpora a su discurso el efecto que este produce en sus interlocutores (ya sean estos sus compañeros, ya sea el profesor) y que ellos le manifiestan por medios tanto lingüísticos como paralingüísticos. (Diccionario de términos clave de ELE, 1997-2017, versión electrónica)

En el presente artículo, enfocamos el feedback como un proceso que complementa a la corrección y, al respecto, nos situamos en la perspectiva de Campbell (1998, p. 84), quien definía este término como la respuesta del profesor o de otro agente externo a la producción escrita del alumno que permitirá que este pueda seguir trabajando en dicha producción con el propósito de perfeccionarla o en palabras de la propia autora: "the response of a teacher, mentor, peer, or some other outside reader to a writer's work, particularly as such response helps facilitate improved writing".

López-Hernández (2009, p. 66), a su vez, asociaba el feedback con una "respuesta al error" afirmando que se trata de un juicio emitido por el profesor, dirigido a la consecución de una corrección lingüística. En esta óptica, el feedback se ha de entender como un mensaje elaborado por el profesor en el que intenta, en primer lugar, destacar "el valor comunicativo de la práctica de los alumnos" (López-Hernández, 2009, p. 71) y, en segundo lugar, transmitir tanto los aciertos como los errores que aparecen en la producción en L2 pretendiendo, por tanto, "responder de forma holística a la práctica del alumno" (López-Hernández, 2009, p. 66).

Las distintas opiniones de profesores e investigadores sobre los beneficios o inconvenientes de proporcionar feedback a las producciones escritas de los alumnos, tanto de L1 como de L2, se han sucedido y siguen sucediéndose a día de hoy. En este sentido, Ferris (2003, p. 14) manifestaba que los comentarios de los profesores pueden ejercer una influencia positiva o negativa en las futuras producciones escritas de los alumnos de manera que: "because teacher is the teacher, any feedback is likely to influence what students do subsequently-and experts disagree as to whether this influence is ultimately helpful or harmful". La autora, venía destacando, desde las décadas de los 70 y los 80, que los comentarios escritos por el profesor en las producciones de los alumnos son considerados como la forma de feedback más utilizada que, sin embargo, se han revelado como poco efectivos, llegando a ser menospreciados y 
calificados como pérdida de tiempo. Sin embargo, a partir de los años 90, comienza a surgir una valoración de los comentarios bien formulados por el profesor, empezando a ser valorados como ayuda para los alumnos.

\section{Conocimientos del alumno y del profesor sobre el error}

Como resultado de la "visión tradicional del error" (Torijano-Pérez, 2004, p. 15) y de la "postura punitiva ante el error escolar" (Torijano-Pérez, 2004, p. 16) los conocimientos que se han transmitido a los alumnos de L2 giran en torno a la idea de sanción y de la "creación de nuevos hábitos" (p. 16). Sin embargo, con la llegada de las nuevas teorías de enseñanzaaprendizaje de una L2, que ofrecen una visión positiva ante el error, se capacita al alumno como conocedor de la importancia que tiene su presencia en las producciones escritas en L2.

Así pues, los conocimientos del alumno han de estar orientados hacia la idea de identificar el error con un "learning step" o "paso de aprendizaje" hacia su L2, aportando una "visión optimista del avance del estudiante" (Torijano-Pérez, 2004, p. 19).

Cassany (1993, p. 18) se acerca a las figuras del profesor y del alumno, con respecto a la corrección, y hace alusión a los roles que corresponden a cada uno de ellos, algo que toda la comunidad educativa entiende y da por hecho. De acuerdo con esto, es el profesor quien asume su papel de corrector mientras los alumnos esperan recibir sus producciones escritas corregidas. En este contexto, se plantea la idea de un modelo dicotómico "activo-pasivo", al que aludía Cassany (1993, p. 18), mediante el cual "el alumno se convierte en mero receptor callado del trabajo del maestro".

Ante esta concepción de los roles establecidos, por parte de los miembros de la comunidad educativa, se impone la necesidad de que estos conozcan y acepten el error como parte del proceso de enseñanza-aprendizaje de la L2. Así pues, Torijano-Pérez (2004) manifestaba que el conocimiento, acerca de los errores, es necesario para el profesor, para el investigador y, sobre todo, para el propio alumno ya que estos son instrumentos tanto de aprendizaje como de enseñanza.

El conocimiento profundo sobre el error, entendido este como "punto de partida en el aprendizaje" (de la Torre, S., Mallart, J., Tort, Ll., Rajadell, N., Laffitte, R. y Oliver, C., 1994, p. 116), ayudará a los alumnos de L2 a mantener una visión de apertura ante este y a percibirlo como síntoma de progreso. Esta percepción evitará el bloqueo y la inhibición del alumno durante el desarrollo de actividades de producción tanto oral como escrita. Los autores mencionados indicaban que el concepto de error debía ser entendido, por el alumno, como un "estímulo de búsqueda y aprendizaje" de su L2. Un "recurso de reflexión por parte de profesores y alumnos" (de la Torre, S., Mallart, J., Tort, Ll., Rajadell, N., Laffitte, R. y Oliver, C., 1994, p. 42) que obliga a ambos a "revisar el proceso y averiguar en qué se ha fallado y por qué".

\section{Aptitud del alumno en torno al error, la corrección y el feedback}

Respecto a la aptitud del alumno ante el error, existe un gran número de autores que defienden la idea de autocorrección de las producciones escritas, especialmente en los niveles avanzados (Cassany, 1993; Constantinescu, 2011; García-Heras, 2004; Guillén-Díaz, 2004; Vez, Guillén y Alario, 2002), a lo que Vez, Guillén y Alario (2002, p. 255) añaden que dicha corrección "requiere en gran medida promover la situación de autoevaluación por parte 
del alumno, junto a la intervención del profesor". Los mencionados autores proponen una "situación de autoevaluación" en la que el alumno cuente con:

Un cuadro de referencias en el que se expliciten los saberes y saber hacer requeridos.

Un código -según el tipo de error- que le permita descubrir sus faltas, pues sabemos que si es el profesor quien ha corregido dando la forma correcta no van a ser tenidas en cuenta, pero así el alumno está atento al error, porque el profesor señala la falta con el código correspondiente al tipo de error y es el alumno quien debe buscar o descubrir el error y corregirlo. Reflexiona sobre las razones de las faltas detectadas. (Vez, Guillén y Alario, 2002, p. 255)

Cassany (1993, p. 16), a su vez, apoyaba la idea de incluir este procedimiento de autocorrección en el aula de manera que se haga sentir al alumno parte del proceso de corrección y, al mismo tiempo, responsable de sus propias producciones escritas. Según el mismo autor, "en raras ocasiones los alumnos tienen la oportunidad de revisarse sus propios errores, de autocorregirse, de reformular sus borradores y mejorar paulatinamente su trabajo", lo cual origina una falta de interés, por parte del alumno, convirtiendo la redacción en algo "lejano a uno mismo, en una obligación escolar".

En la misma línea, García-Heras (2004, p. 65) reflexionaba sobre la importancia de involucrar al alumno en el proceso de escritura creando un hábito de autocorrección y una "actitud activa". La mencionada autora, afirmaba que, si los alumnos "se involucran intelectualmente en la actividad, es mucho más probable que aprendan de su propia experiencia".

Por su parte, Ferris (2003) proponía el uso de un modelo de autoevaluación por parte de los alumnos de L2. En su opinión, para conseguir que estos lleguen a alcanzar un nivel adecuado de competencia en expresión escrita, se impone que el profesor, proporcione el feedback correspondiente junto con una intervención o respuesta por parte de un compañero de clase, seguida de una autoevaluación guiada. Los tres elementos indicados constituyen una parte fundamental de la corrección de errores por lo que, como profesores de L2, somos responsables de crear situaciones de aprendizaje en las que se incluyan los mencionados elementos. Además de servir de ayuda al alumno, a la hora de enfrentarse ante una actividad de producción escrita en L2, esta combinación de tareas le aportará un grado de seguridad y confianza en sí mismo, que se podría convertir en un gran estímulo de cara a futuras producciones escritas.

\section{Actitud del alumno ante el error, la corrección y el feedback}

Hablar de actitudes implica acercarnos a un "conjunto de representaciones mentales (creencias, valores, prejuicios) y sus manifestaciones en forma de opiniones y hábitos o normas de conducta" (Cassany, 2009, p. 56). El tema de las actitudes, en general, y de los profesores, en particular, ha captado la atención de numerosos especialistas en Psicología y Didáctica. Sin embargo, en lo que concierne a la actitud orientada al alumno, a pesar de su valor, no se le ha concedido la atención que merece.

La idea de que el alumno debe ser consciente de los errores cometidos en sus producciones tanto orales, como escritas, y aceptarlos como "motor de aprendizaje" es compartida por varios autores entre los cuales mencionamos a Giovannini, Martín-Peris, Rodríguez-Castilla y Simón-Blanco (1996, p. 12).

Santos-Gargallo y Alexopoulou (2014, p. 431) definen las actitudes de los alumnos como un "concepto un tanto impreciso" ya que "son un constructo difícil de delimitar por su fuerte carga de subjetividad". Las autoras plantean la necesidad de analizar los factores 
individuales que intervienen a lo largo del proceso de aprendizaje de una L2 y de "reflexionar sobre las creencias, las actitudes con las que los alumnos llegan a clase" (Santos-Gargallo y Alexopoulou, 2014, p. 430). Se trata de creencias entendidas como representaciones mentales, las cuales: "son recursos cognitivos conformados por concepciones preestablecidas que posee el ser humano para organizar describir e interpretar el mundo y las experiencias que lo rodean. Constituyen una herramienta para procesar la información y funcionan como una guía de actuación" (Santos-Gargallo y Alexopoulou, 2014, pp. 430-431).

Desde el punto de vista de Santos-Gargallo y Alexopoulou (2014, p. 445), las creencias de los alumnos griegos que estudian ELE varían en función del nivel de lengua que poseen. Asimismo, las citadas autoras, tomando como referencia los niveles del Marco Común Europeo de Referencia para las Lenguas: aprendizaje, enseñanza, evaluación (MCER) (2002), observaban que: "en el nivel A2 los alumnos consideran el error como algo positivo, mientras que en el nivel C1 predomina la consideración negativa" (Santos-Gargallo y Alexopoulou, 2014, p. 445). A partir de esta afirmación, podemos manifestar que "en los primeros estadios, los errores son vistos como signo de desarrollo del proceso, sin embargo, en un nivel más avanzado son considerados objeto de frustración en el dominio de la lengua y en el desarrollo de su competencia comunicativa" (Santos-Gargallo y Alexopoulou, 2014, p. 445). Destacamos, pues, esta relación entre nivel de competencia lingüística y valoración de la corrección. Los alumnos con un nivel de L2 elevado muestran una valoración del error que suele manifestarse como negativa ya que lo consideran símbolo de frustración. Sin embargo, los alumnos con un nivel de L2 básico no experimentan dicho sentimiento y tienden a valorar el error de una forma positiva, relacionándolo con una muestra de aprendizaje (Merino Mañueco, 2016).

Por su parte, desde el ámbito de las matemáticas, Rach, Ufer y Heinze (2013, p. 28), desde el contexto educativo alemán, exponían, con respecto al tema del error, que los alumnos no están acostumbrados a reflexionar sobre sus propios procesos de aprendizaje ni sobre sus propios errores. Podríamos trasladar esta idea al ámbito de la enseñanza-aprendizaje de L2 y afirmar que, generalmente son pocas las reflexiones del alumno hacia su propio proceso de aprendizaje de L2.

Ya en el año 1996, Truscott ponía de manifiesto la relación entre un aprendizaje eficaz y un estado de tensión física o emocional que puede llegar a incidir negativamente en el aprendizaje. Desde el punto de vista del autor, el éxito o el fracaso en el proceso de aprendizaje depende directamente de la cantidad de estrés del alumno. Solo cuando este se encuentre tranquilo y relajado conseguirá alcanzar un aprendizaje significativo de la L2. En este sentido, el autor deja clara su posición en contra de la corrección y del feedback en las producciones escritas.

De acuerdo con Leki (1990), existe una diferencia entre la enseñanza de L1 y la de L2, con respecto a las actitudes de los alumnos ante las correcciones y las marcas del profesor. La citada autora sostiene la idea de que los estudiantes de L1 no valoran los comentarios o correcciones que el profesor incluye en sus producciones escritas, considerando que no aportan información relevante que pueda servirles de ayuda. Sin embargo, los estudiantes de L2, no solo demandan una corrección en la que todos los errores aparezcan marcados, sino que, además, reclaman la incorporación de comentarios del propio profesor, aportando pistas que les permitan corregir sus propios errores.

Así es como, Ferris (2003), mostrando su acuerdo con Leki (1990), no solo pone de manifiesto la demanda por parte del alumno de L2, de ser corregido, sino también de recibir el feedback correspondiente. Desde esta misma consideración, manifestaba su desacuerdo con 
Truscott (1996), respecto a su propuesta de apartar a los alumnos de L2 de la corrección y del feedback del profesor ya que, en su opinión, dichos alumnos, en general, defienden el uso de estos, por su gran utilidad en el proceso de enseñanza-aprendizaje.

Ferris (2003) sugiere a los profesores la elaboración de encuestas a partir de las cuales los alumnos puedan proporcionar información relacionada con sus preferencias en cuanto a la forma de corrección de errores y al tipo de feedback que les interesa recibir por parte del profesor. La autora propone esta actividad como un medio a través del cual se podrá recabar información relativa a las opiniones de los alumnos y sus posibles reacciones en torno a la práctica docente. Por lo tanto, como profesores, se impone la necesidad de preguntar a los alumnos acerca de sus preferencias en torno al tipo de corrección y feedback, lo cual permitirá desarrollar actitudes positivas hacia el aprendizaje, aumentar su motivación y estimular el acercamiento entre profesores y alumnos.

Refiriéndose a las actitudes de los alumnos hacia el aprendizaje de su L2, Gardner (2010) mencionaba el importante papel que juega la relación profesor-alumno en este. Así es como, insiste en la idea de que la personalidad del profesor, su comportamiento y los métodos utilizados en el aula serán determinantes a la hora de establecer dicha relación. En definitiva, el nivel de compromiso del profesor en su trabajo diario, la forma de dirigirse a los alumnos, su cordialidad y, sobre todo, el grado de confianza mostrado se manifiestan como aspectos que influirán de una manera decisiva en las actitudes de los alumnos hacia su L2.

Otro de los aspectos que el profesor debe tener en cuenta cuando se enfrenta a una corrección de errores es la motivación; entendida por Gardner (2010, p. 186) como una parte intrínseca del proceso de enseñanza-aprendizaje de una L2. En este acercamiento, el mencionado autor señalaba la necesidad de una relación de cercanía y respeto entre profesor y alumno que motive al alumno a intentar alcanzar el éxito educativo en el aula de L2 puesto que: "when students are treated well, respected and encouraged they feel that their work is meaningful and as a consequence they will develop high levels of performance".

\section{Conclusiones}

En el presente trabajo nos hemos acercado a la enseñanza-aprendizaje de la expresión escrita en L2. Consideramos que es de suma importancia desde el punto de vista didáctico que el alumno elabore sus producciones escritas de una manera organizada, utilizando un vocabulario rico y matizado. Consecuentemente, se impone que el profesor siempre procure introducir ejercicios que motiven al alumno para que este mantenga una actitud positiva hacia el aprendizaje de la L2, utilizando una metodología que favorezca dicha actitud positiva hacia la lengua y hacia la elaboración de producciones escritas. En el aula de lengua en general y en el de L2, en nuestro caso particular, se impone como necesidad enseñar todas aquellas estrategias y recursos didácticos que permitan desarrollar la expresión escrita como destreza. Para ello, el profesor deberá solicitar al alumno que produzca una amplia variedad de textos escritos siempre relacionados con temas cercanos a sus intereses particulares.

Además de esto, se impone como condición intrínseca la presencia de un adecuado tratamiento de errores entendido desde la perspectiva de una corrección y un feedback en el que el profesor muestre una actitud positiva. Todo lo cual permitirá que anime a los alumnos a expresarse de una manera libre sin temor a cometer errores. Desde este punto de vista se revela como necesidad la presencia de una formación del profesorado de L2 en torno al error y a su corrección. 
La noción de tratamiento del error se sintetiza en una serie de estrategias y técnicas en las que se toma como punto de partida el error como herramienta de aprendizaje en el aula de L2 por cuanto que, para poder llegar a contribuir desde el área de enseñanza-aprendizaje de L2, a optimizar el tratamiento del error se impone la necesidad de una toma de conciencia de los errores tanto por parte del profesor como del alumno. En todo este acercamiento, se ha hecho evidente la idea de que es nuestro deber como profesores desarrollar ese tipo de estrategias en el alumno como parte del tratamiento del error en el aula de L2 haciendo uso de distintos recursos como: cuadernos de superación de errores, grabaciones, actividades de ejercitación, actividades lúdicas, etc.

\section{Bibliografía}

Campbell, C. (1998). Teaching Second-Language Writing: Interacting with a Text. Scarborough: Heinle \& Heinle.

Cassany, D. (1993). Reparar la escritura. Didáctica de la corrección de lo escrito. Barcelona: Graó.

Cassany, D. (2009). La composición escrita en E/LE. Didáctica del Español como Lengua Extranjera. Expolingua. 9, 47-66.

Centro Virtual Cervantes (1997-2017). Feedback>Retroalimentación. Diccionario de Términos Clave de ELE (versión electrónica). Recuperado de http://cvc.cervantes.es/ensenanza/ biblioteca_ele/diccio_ele/diccionario/

Constantinescu, I. (2011). Dictionnaire pratique de didactique du français langue étrangère (F.L.E). Bucureşti: Fundaţia România de Mâine.

d'Aquino, A. (2016). ¡De los errores se aprende! La corrección como instrumento didáctico. Textos. Didáctica de la Lengua y de la Literatura. 71, 7-13.

de la Torre, S., Mallart, J., Tort, Ll., Rajadell, N., Laffitte, R. y Oliver, C. (1994). Errores y currículo. Tratamiento didáctico de los errores en la enseñanza. Barcelona: Promociones y publicaciones universitarias.

Dumbrăvescu, D. G. (2017). Los manuales escolares y la formación inicial del profesorado de ELE. Valencia: Educalia.

Durán, R. (2011). Guidelines for Error Correction in the EFL Classroom. En S. House (Coord.). Inglés. Investigación, innovación y buenas prácticas. (pp. 61- 67). Barcelona: Graó.

Fathman, A. K. y Whalley, E. (1990). Teacher response to student writing: focus on form versus content. En B. Kroll (Ed.). Second Language Writing. Research insights for the classroom. (pp. 178-190). Cambridge: Cambridge University Press.

Fernández-López, S. (1995). Errores e interlengua en el aprendizaje del español como lengua extranjera. Didáctica. 7, 203-216.

Fernández, S. (1997). Interlengua y análisis de errores en el aprendizaje del español como lengua extranjera. Madrid: Edelsa.

Ferris, D. y Hedgcock, J. (1998). Teaching ESL composition: Purpose, process, and practice. Mahwah, NJ: Lawrence Erlbaum Associates.

Ferris, D. (2003). Response to Student Writing: Implications for Second Language Students. Mahwah, NJ.: Routledge. 
García-Heras Muñoz, A. (2004). Lingüística y enseñanza: el tratamiento de los errores en la enseñanza del inglés como lengua extranjera. Docencia e investigación: revista de la Escuela Universitaria de Magisterio de Toledo. 14, 49-70.

Gardner, R. C. (2010). Motivation and second language acquisition: The socio-educational model. New York, NY.: Peter Lang.

Gascoigne, C. (2004). Examining the Effect of Feedback in Beginning L2 Composition. Foreign Language Annals. 37 (1), 71-76.

Giovannini, A. Martín-Peris, E., Rodríguez-Castilla, M., y Simón-Blanco, T. (1996). Profesor en Acción. (Vol. 2). Madrid: Edelsa.

Guillén-Díaz, C. y Castro-Prieto, P. (1998). Manual de autoformación para una didáctica de la lengua-cultura extranjera. Madrid: La Muralla.

Guillén-Díaz, C. (2004). Error. En F. Salvador-Mata, J. L. Rodríguez-Diéguez y A. Bolívar-Botía (Dirs.). Diccionario Enciclopédico de Didáctica. (Vol. 1). (p. 627). Málaga: Aljibe.

Jericó, P. (9 de julio de 2013). Cómo dar un buen “feedback”. El País. Recuperado de https:// elpais.com/elpais/2013/07/09/laboratorio_de_felicidad/1373353794_137335.html

Jiménez-González, J. E. y Muñetón-Ayala, A. (2002). Dificultades de aprendizaje de la escritura . Aplicaciones de la psicolingüística y de las nuevas tecnologías. Madrid: Trotta.

Leki, I. (1990). Coaching from the margins: issues in written response. En B. Kroll (Ed.). Second Language Writing. Research insights for the classroom. (pp. 57-68). Cambridge: Cambridge University Press.

López-Hernández, A. (2009). Feedback y corrección de errores en el aula de L2 (I): actividades orales. Educación y futuro: revista de investigación aplicada y experiencias educativas. 20, 65-78. Recuperado de https://dialnet.unirioja.es/servlet/articulo?codigo=3233775

Merino Mañueco, S. (2016). El análisis de errores en los enfoques actuales de enseñanzaaprendizaje de segundas lenguas. En M. á. Lamolda González (Comp.). La formación y competencias del profesorado de ELE. Actas del XXVI Congreso Internacional ASELE. (pp. 693-698). Granada: Universidad de Granada.

Ministerio de Educación, Cultura y Deporte. (2002). Marco Común Europeo de referencia para las lenguas: aprendizaje, enseñanza, evaluación. Madrid: Secretaría General Técnica del MECD y Grupo Anaya.

Pawlak, M. (2014). Error correction in the foreign language classroom. Reconsidering the issues. Second Language Learning and Teaching Series. Berlin. Heidelberg: SpringerVerlag. doi: 10.1007/978-3-642-38436-3_4

Rach, S., Ufer, S. y Heinze, A. (2013). Learning from errors: effects of teachers training on students' attitudes towards and their individual use of errors. PNA. 8 (1), 21-30.

Roy, D. (1991). Les enseignants et les enseignantes exercent-ils une réelle influence sur l'apprentissage au collégial? Pédagogie collégiale. 5 (1), 37-40.

Santos-Gargallo, I. y Alexopoulou, A. (2014). Creencias y actitudes de profesores y alumnos griegos de español ante las técnicas de corrección en la interacción oral: estudio comparativo intragrupos. Didáctica. Lengua y Literatura, 26, 429-446. 
Torijano-Pérez, J.A. (2004). Errores de aprendizaje, aprendizaje de los errores. Madrid: Arco Libros.

Truscott, J. (1996). Review Article. The Case Against Grammar Correction in L2 Writing Classes. Language Learning. 46 (2), 327-369.

Vargas-Venegas, G. (2008). Desarrollo de la fluidez oral en ELE mediante los periódicos, las canciones, las películas y los juegos. (Memoria de Máster en Formación de profesores de español como lengua extranjera). Universidad de León en colaboración con la Fundación Universitaria Iberoamericana (FUNIBER). Recuperado de https:// www.mecd.gob.es/dam/jcr:10d0a665-9f07-4c61-927d-7b4681ed482c/2010-bv-1121vargas-pdf.pdf

Vez, J. M. (Ed.), Guillén, C. y Alario, C. (2002). Didáctica de la lengua extranjera en educación infantil y primaria. Madrid: Síntesis.

Zamel, V. (1985). Responding to Student Writing. TESOL Quarterly. 19 (1), 79-101. doi:10.2307/3586773. 
